\title{
A new model for the prediction of track sound radiation
}

\author{
Xianying Zhang, David J Thompson, Giacomo Squicciarini \\ Institute of Sound and Vibration Research, University of Southampton, \\ Southampton, SO17 1BJ, UK \\ E-mail: xianyingzhang96@gmail.com
}

\begin{abstract}
Summary
The TWINS model is a widely used and well-established model for rolling noise which has been validated against field measurements in terms of overall noise spectra and levels. However, there are still some areas that can be improved. In particular, the radiation from the rail is based on a model of a rail in free space and there are also limitations in the model for the sound radiation from the sleepers. This paper draws on recent research into the effects of the proximity of the rail and sleeper to an absorbing ground on their sound radiation. Moreover, the ballast is acoustically absorbing to some extent because of the gaps between the ballast particles and this can affect the noise radiation by the rail and sleeper. The ballast absorption is represented here using the Johnson-Allard model with measured values of flow resistivity and porosity. Additionally, the Delany and Bazley model is introduced with a higher value of flow resistivity for comparison. These are used to produce the normal impedance of the ballast layer which is introduced in boundary element calculations of the radiation from the rail and sleepers. Comparisons are made first with the sound radiation from a 1:5 scale track model which has been measured reciprocally in the reverberation chamber. It is shown that the impedance using the measured flow resistivity is inadequate for use in the numerical models; probably an extended reaction model would be more appropriate. However, the Delany and Bazley model with the higher flow resistivity gives better agreement with the measurements and is a practical solution. The new models have also been used together with TWINS to predict the sound radiation from an operational track and the results have been compared with an example field measurement. The new models are found to give an improvement at low frequencies, where the sleeper is the dominant noise source.
\end{abstract}

\section{Introduction}

The most important source of railway noise in most situations is rolling noise, which is caused by wheel and rail vibration induced at the wheel/rail contact area. The TWINS model [1,2] is well-established as a method of predicting the generation of vibration and noise from the railway wheels and track and this has been used successfully to design noise mitigation measures. However, there exist some limitations in this model. In particular, the sound radiation from the rails is calculated under the simplifying assumption that they radiate into free space, whereas in the reality they are located close to the ground. There are also limitations in the way that the sleepers are treated in TWINS. They are assumed to be embedded in a rigid ground and the effect of multiple sleepers is taken into account in a heuristic way [3]. Recently, the authors have developed improved models for the sound radiation from rails in proximity to an absorptive ground using the boundary element method (BEM) and validated them against laboratory experiments [4]. In [5], improved boundary element models for the sound radiation from sleepers have been presented and validated through laboratory measurements for an absorptive ground consisting of melamine foam.

The ballast is also an important component of the track. Railway ballast is a porous medium with absorptive properties which may modify the sound radiation of the rail and the sleeper. Therefore, it is necessary to take these boundary conditions into account in the models for the sound radiation from the track. In this paper, measurements of ballast absorption are presented and two different models are used to represent this. The effects on the sound radiation from the rail and the sleepers are predicted by the boundary element method. Then, the various models are combined to give predictions of the sound radiation from the complete track. Results are shown first for a 1:5 scale track and are compared with corresponding experimental results obtained in the laboratory. Finally, the sound radiation from a full size track is predicted by using the new models together with the TWINS software and comparisons are made with existing field measurements. 


\section{Ballast absorption and its effects on the track sound radiation}

\subsection{Ballast absorption}

Measurements of the absorption of railway ballast obtained in a reverberant chamber have been presented by Broadbent et al [6]. In that work the results were fitted to a multiple layer model with flow resistivities between 25 and $51 \mathrm{kPa} . \mathrm{s} / \mathrm{m}^{2}$. However, in [7] the flow resistivity of railway ballast was measured as $200 \mathrm{~Pa} . \mathrm{s} / \mathrm{m}^{2}$.

Due to the difficulties of working with full scale ballast (typical stone sizes are $50 \mathrm{~mm}$ ) measurements have been made here of the flow resistivity and porosity of samples of 1:5 scale ballast with the correct gradation [8] at reduced scale. The flow resistivity was measured by passing air through a tube of internal diameter $146 \mathrm{~mm}$ filled with ballast samples of 300 and $600 \mathrm{~mm}$ thickness. The mean value was found to be $280 \pm 20 \mathrm{~Pa} . \mathrm{s} / \mathrm{m}^{2}$. In addition the porosity was determined by adding a measured amount of water to a bucket filled with scale ballast and a value of 0.463 was found. These properties of the 1:5 scale ballast are similar to the values for full scale ballast given in [7] but are believed to be more reliable as a correspondingly larger sample of ballast could be included in the test rigs. The absorption coefficient of this reduced scale ballast was also measured in a reverberation chamber [9], as shown in Figure 1. This is comparable to the result for the equivalent full scale sample from [6], shown in Figure 2.

The Johnson-Allard model $[10,11]$ has then been used with the above parameter values to obtain the normal acoustic impedance. The values of tortuosity and characteristic lengths were chosen to give a good fit to the measured absorption coefficient. This gave a tortuosity of 1.3 and viscous characteristic length of $0.482 \mathrm{~mm}$ for the 1:5 scale ballast and values of 1.0 and $1.63 \mathrm{~mm}$ for full scale ballast. The results are compared with the corresponding measured diffuse field absorption coefficients in Figure 1 and Figure 2. Good agreement can be seen between them for both the 1:5 scale and full size ballast.

However, for propagation at grazing incidence above a layer of ballast, the Delany and Bazley model [12] for an infinite layer with a flow resistivity of $50 \mathrm{kPa} . \mathrm{s} / \mathrm{m}^{2}$ has been found to give reasonable predictions [13]. This model will be also used as an alternative to study the influence of the ballast absorption in this paper, even though the flow resistivity used in the model $\left(50 \mathrm{kPa} . \mathrm{s} / \mathrm{m}^{2}\right)$ is quite different from the measured value $(280$ $\mathrm{Pa} . \mathrm{s} / \mathrm{m}^{2}$ ). By using this model, the corresponding absorption coefficients in a diffuse field for the two types of the ballast are also presented in Figure 1 and Figure 2. The agreement between the predictions and the measured results for both cases is much worse than for the Johnson-Allard model.

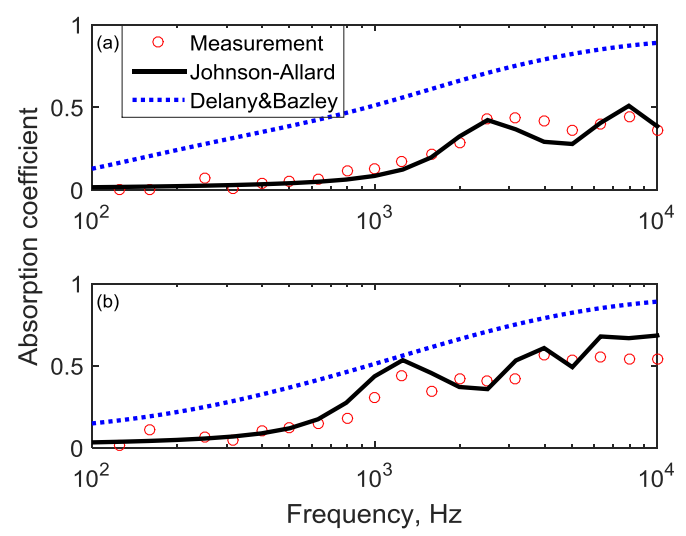

Figure 1 Absorption coefficient of the 1:5 scale ballast. Comparisons between reverberation chamber measurements and prediction. (a) Thickness $0.03 \mathrm{~m}$; (b) Thickness $0.06 \mathrm{~m}$

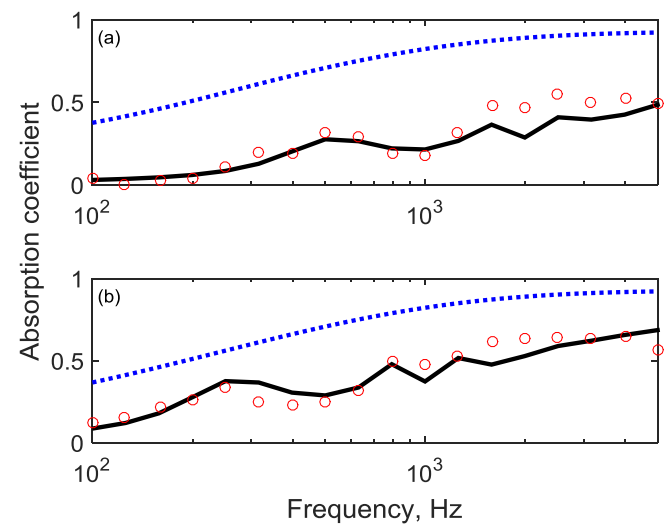

Figure 2 Absorption coefficient of the full scale ballast. Comparisons between the measured results from [8] and predictions. (a) Thickness $0.17 \mathrm{~m}$; (b) Thickness $0.33 \mathrm{~m}$

\subsection{Rail and sleeper radiation}

Two-dimensional boundary element calculations have been carried out for a full scale rail located $50 \mathrm{~mm}$ above a layer of ballast using both impedance models described above. In the Johnson-Allard model the thickness of the ballast layer is set to $500 \mathrm{~mm}$, whereas in the Delany and Bazley model it is neglected. The radiation ratio of the rail is presented in Figure 3, for purely vertical and lateral vibration of the rail. Compared with the result for 
a rail in free space, as used in TWINS, dips occur below $1 \mathrm{kHz}$ in the results predicted by the Johnson-Allard model with the measured flow resistivity, which are due to the effects of the layer thickness in the impedance. When the Delany and Bazley model with the higher flow resistivity is used, at low frequency the radiation ratio increases for lateral motion, but the radiation ratio for vertical motion reduces, similar to results found for a rigid ground [3]. For both the vertical and lateral motion of the rail, at high frequency the radiation ratio is reduced by around $2 \mathrm{~dB}$ because of the absorption of the ballast in either model.

For the sleepers, a three-dimensional boundary element model is used to assess the effect of the ballast absorption on the sound radiation. Three sleepers are included in the model, which are assumed to be vibrating with amplitudes of $0.5,1$ and 0.5 to represent the relative vibration transmitted by the rail excited at a point above the central sleeper [5]. The sleepers are assumed to be fully embedded in the ballast so only their upper surface is visible. Figure 4 shows the radiation ratio obtained using the different models of ballast impedance. These are compared with the result obtained from the model currently implemented in TWINS. The latter is an approximation based on the radiation from a rectangular piston set in an infinite baffle with a heuristic correction for the presence of multiple sleepers [4]. The predicted radiation ratio from the sleeper in the current work has allowed for this effect in a more rigorous way. Dips again occur below $1 \mathrm{kHz}$ for the case based on the Johnson-Allard model with the measured flow resistivity.

Although not shown here, measurements on individual 1:5 scale rails and sleepers together with the scale ballast indicate that the results obtained with the Delany \& Bazley model with flow resistivity of $50 \mathrm{kPa} . \mathrm{s} / \mathrm{m}^{2}$ are more consistent with the measured data.

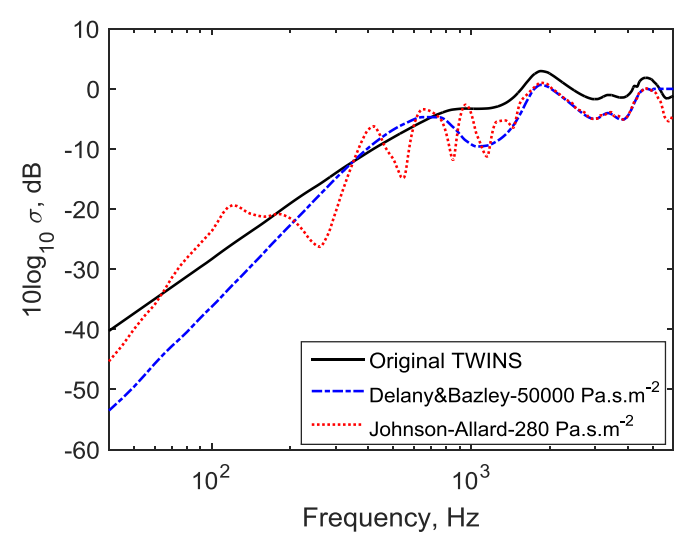

(a) Vertical motion

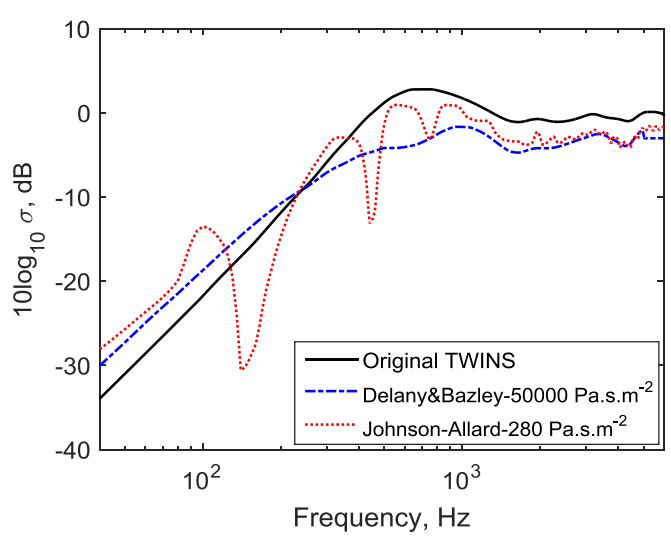

(b) Lateral motion

Figure 3 Comparison of the results of different models for the radiation ratio of a full size rail

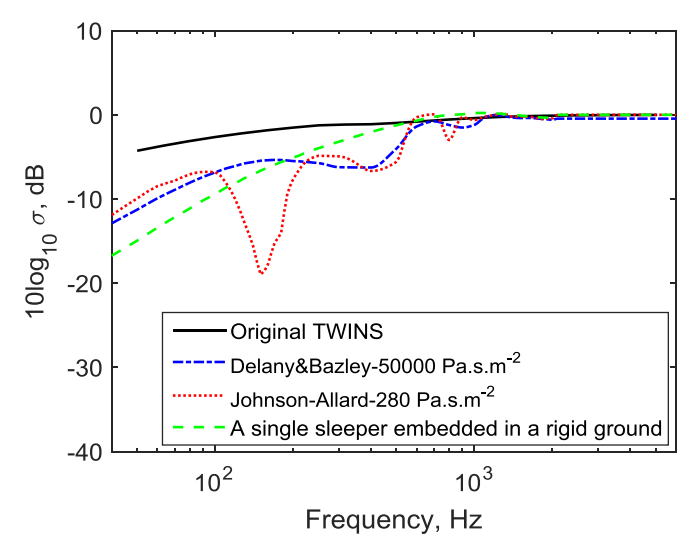

Figure 4 Comparison of the results of different models for the radiation ratio of the full size sleepers

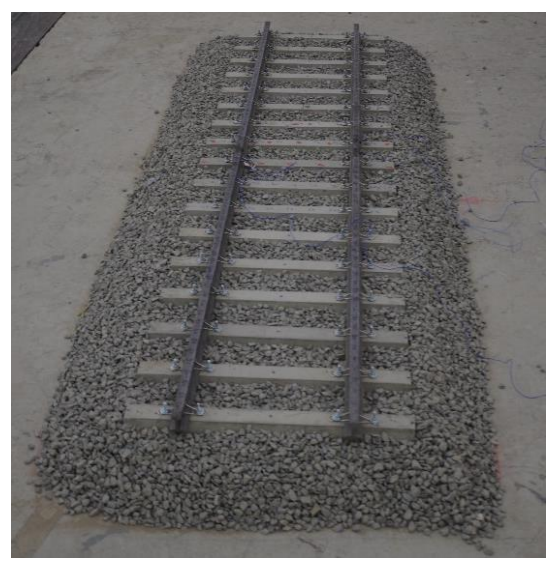

Figure 5 Experimental set up for the 1:5 scale whole track embedded in ballast 


\section{Validation using 1:5 scale track}

Validation measurements have been carried out using a 1:5 scale model track. This consisted of two $2 \mathrm{~m}$-long rails attached via spring clips to 17 concrete sleepers. These were arranged with a spacing of $0.12 \mathrm{~m}$ (corresponding to a full scale distance of $0.6 \mathrm{~m}$ ). The track was embedded in 1:5 scale ballast, with overall dimensions $2 \mathrm{~m} \times 0.8 \mathrm{~m} \times 100 \mathrm{~mm}$, located on the floor of a large reverberation chamber; the depth of ballast beneath the sleepers was $60 \mathrm{~mm}$. The corresponding set up is shown in Figure 5. Rubber rail pads are also located between the rail foot and the sleepers. These consisted of pieces of neoprene with a thickness of $2.5 \mathrm{~mm}$ and dimensions $45 \mathrm{~mm} \times 35 \mathrm{~mm}$. The average stiffness of the railpads has been determined to be $15 \mathrm{MN} / \mathrm{m}$.

\subsection{Point mobility and decay rate}

The driving point mobility of this scale model track is presented in Figure 6. Comparison is also made with the corresponding predictions obtained by using an analytical track model, based on a continuously supported infinite Timoshenko beam [14, 15]. Quite good agreement can be seen between the predictions and the measured point mobility, indicating that the track behaves in a similar way to a full size track. The fluctuations in the measurements at high frequencies are due to reflections from the finite length of the rail.

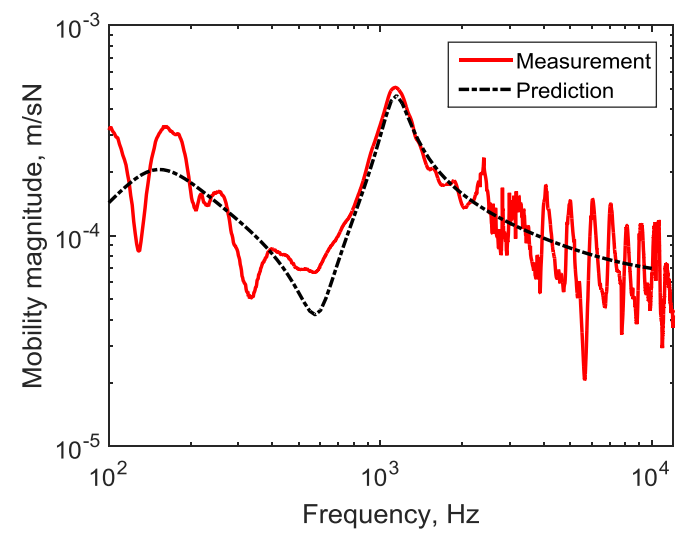

(a) Magnitude

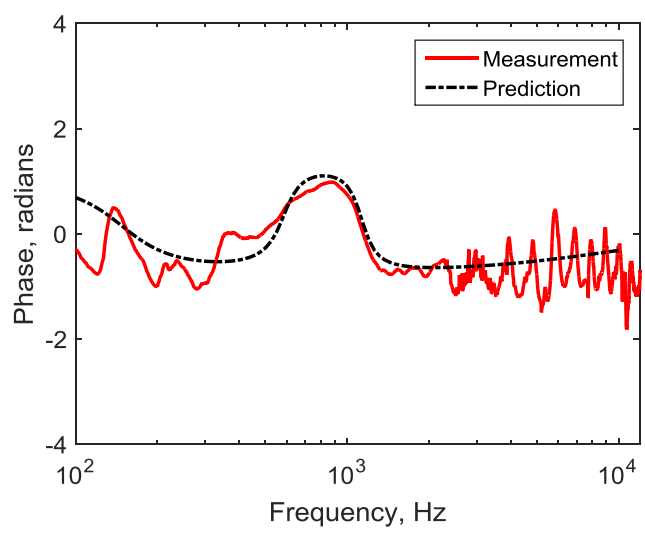

(b) Phase

Figure 6 Point mobility of the 1:5 scale track. Rail pad stiffness $15 \mathrm{MN} / \mathrm{m}$, loss factor 0.2. Ballast stiffness 1.0 MN/m per half-sleeper, loss factor 1.0

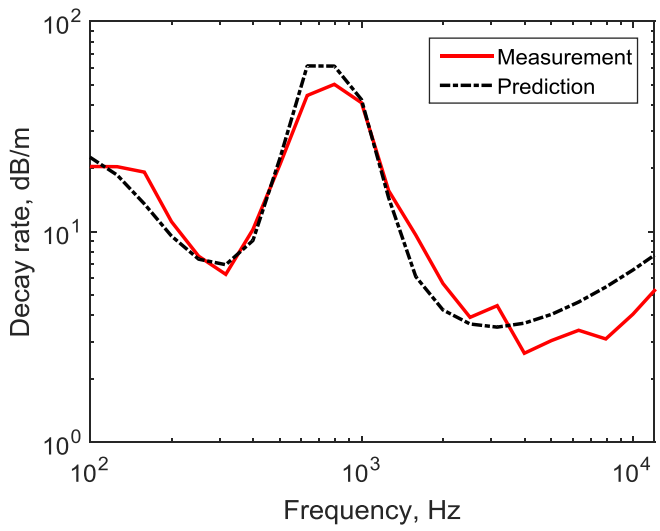

Figure 7 Decay rate for an equivalent infinite track for the 1:5 scale track

Measurements based on the method from EN 15461 [16] have also been made to verify that the decay rate for the 1:5 scale track is representative of a real track. In applying this method, the initial point was set at one end of the track and the mobility was measured at 30 positions by moving the impact hammer. Corrections were made 
for the finite length of the track in processing these measured results [17]. The results are shown in Figure 7 and compared with the results of the infinite track model [14, 15]. Good agreement can be seen between the predicted decay rate and the measured one. The decay rate has a typical form, with a high value at low frequency, a peak in the region where the sleeper acts as a dynamic absorber, a sudden drop above the resonance of the rail on the pad before rising again at high frequency. Thus, it can be concluded that the 1:5 scale track is a good representation of a real track.

\subsection{Sound power from the whole track}

The sound power $W$ radiated by a vibrating object can be written as [18]

$W=\rho_{0} c_{0} S\left\langle\overline{v^{2}}\right\rangle \sigma$

where $\rho_{0}$ is the density of air, $c_{0}$ is the speed of sound, $S$ is the surface area of the vibrating structure and $\left\langle\overline{v^{2}}\right\rangle$ is the surface-averaged mean-square velocity normal to the surface in the frequency band of interest. The parameter $\sigma$ is the radiation ratio or radiation efficiency.

The mean-square velocity of the rails and sleepers for a unit force on the railhead has been measured by applying a reciprocal method. The transfer mobility was measured for vertical excitation at 69 points along each rail (4 points spaced equally along the rail section in each sleeper span), 11 points spaced equally along the central three sleepers, and 3 points spaced equally along all the other sleepers. Two accelerometers were located vertically at the centre of the railhead above the central sleeper, one on each rail. By reciprocity these measurements represent the transfer mobility from a force at the railhead at the centre of the rail to the velocity of the rail or the sleeper.

The sound power from the whole track for a unit force applied at the rail has been measured reciprocally in a reverberation chamber. Comparison is made with the sound power from both the rails and the sleepers determined by using the corresponding measured vibration and the predicted radiation ratios, including the effect of the ballast absorption. The radiation ratio of the rails is estimated by using a weighted average of the results for a rail attached to a rigid ground [3] and a rail $20 \mathrm{~mm}$ above the ballast. To estimate the sound power from the sleepers, the radiation ratio is based on three sleepers with vibration amplitudes in the ratio of 0.5:1:0.5, as shown in Figure 4 for the full size sleepers.

Figure 8 presents the measured sound power from the assembly normalised by the mean square force. The results are shown in 1/12 octave bands. These are compared with the estimated sound power from the two rails and 17 sleepers based on their measured vibration. As can be seen, the sound power from the sleepers dominates the noise up to $2000 \mathrm{~Hz}$ ( $400 \mathrm{~Hz}$ at full size) as the rail pads reduce the vibration transmission from the rails to the sleepers at higher frequencies, where the rail dominates.

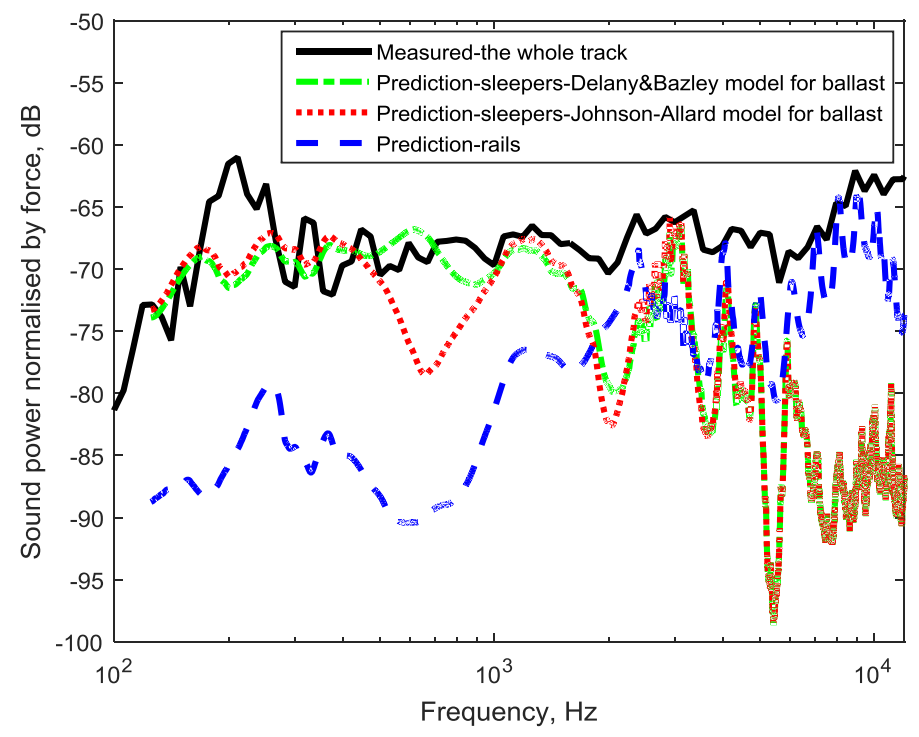

Figure 8 Comparison of measurements and predictions for the sound power of the scale model track shown in Figure 5. 
The radiation ratio of the sleepers embedded in ballast obtained with the Delany and Bazley model gives a better prediction of their sound power than that predicted using the Johnson-Allard model, especially in the frequency range $500 \sim 1000 \mathrm{~Hz}$. This discrepancy is related to the dips in the predicted radiation ratio of the sleepers embedded in ballast for the Johnson-Allard model, as shown in Figure 4. This suggests that the impedance obtained with the measured flow resistivity is not suitable for use in the BEM model, even though good agreement was found with the ballast absorption measured in a diffuse field, as presented in Figure 1. This is likely to be due to limitations in the assumption of local reaction, implied in the use of an impedance boundary condition in BEM, whereas an extended reaction model is more appropriate [13].

\section{Application to operational track}

Finally the current models for the sound radiation from the rail and sleeper are applied to an operational track. The noise from the track is predicted using the TWINS software which is modified to include the results of the new radiation models. These predictions are compared with existing measurements obtained at Fishbourne, UK [19]. The sound pressure spectrum was measured at a point $7.5 \mathrm{~m}$ away from the track and $1.5 \mathrm{~m}$ above the ground during the passage of Class 377 Electrostar EMUs at a speed of $118 \mathrm{~km} / \mathrm{h}$. The rail roughness and track decay rates were measured at the site and wheel roughness measurements were available from similar trains [19]. The pad stiffness was estimated as $120 \mathrm{MN} / \mathrm{m}$ for the vertical direction and $13 \mathrm{MN} / \mathrm{m}$ for the lateral direction. Results will be shown from the existing TWINS software and additionally two modified predictions including the effects of the ground on the sound radiation from the rail and sleeper, as shown in Figure 3 and Figure 4.

\subsection{Prediction of the sound radiation from different components in the track}

The component of the sound pressure level from the sleepers at the microphone position is presented in Figure 9. Results are shown for the original model and the updated models. As expected from the radiation ratios shown in Figure 4, the contribution from the sleepers obtained by using the current models is lower than that obtained based on the original TWINS model. A strong dip can be seen at $160 \mathrm{~Hz}$ for the case based on the Johnson-Allard model due to the thickness effect.

The predicted sound pressure component from the rails at the microphone position is shown in Figure 10. Results are again shown for the original model and the updated models. As can be seen, there are considerable differences between the results of these models at low frequency. However, in this frequency region the sleeper is actually the dominant source. Hence, although the contribution from the rails is obviously modified by the current models at low frequency, this difference will not affect the predicted total noise for a ballasted track such as this.

The sound pressure component from the wheels is shown in Figure 11. This is unaffected by the changes introduced here. As can be seen by comparison with the other components, the wheel will become the dominant source at high frequencies, above about $2 \mathrm{kHz}$.

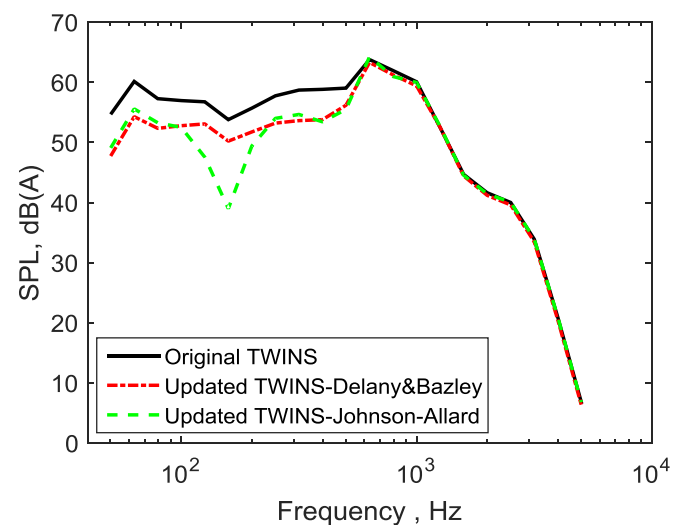

Figure 9 Predicted A-weighted sound pressure level of the component from the sleepers

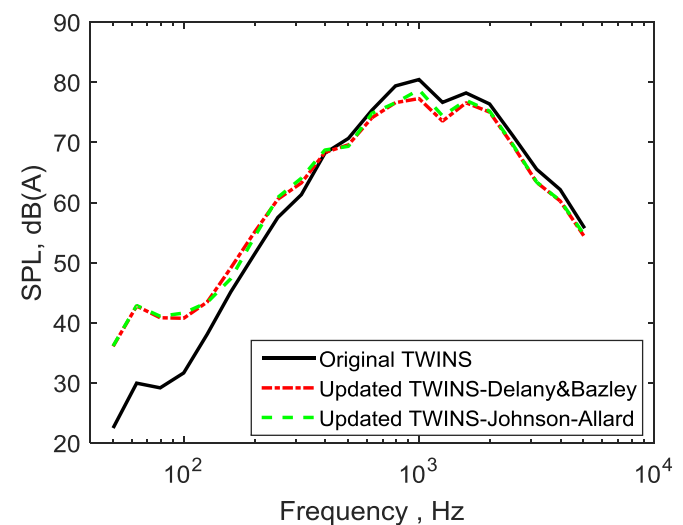

Figure 10 Predicted A-weighted sound pressure level of the component from the rails 


\subsection{Prediction of the sound radiation from the whole track}

The measured and predicted total A-weighted sound pressure levels are shown in one-third octave bands in Figure 12. Good agreement can be seen between the measurements and the predictions from both the original and updated versions of TWINS, especially bearing in mind that the measured results are subject to a variation of $+/-3 \mathrm{~dB}(\mathrm{~A})$ in each $1 / 3$ octave band due to variations in train speed, wheel roughness and temperature [19]. The new radiation ratios have a significant effect below $200 \mathrm{~Hz}$, and in the current example show improved agreement with the measurements. The large dip at $125 \mathrm{~Hz}$ in the results based on the measured flow resistivity of the ballast is not consistent with the measurements whereas the results for the higher value of flow resistivity show better agreement. The differences between the models are smaller at higher frequencies, with a small reduction seen in the rail component between 800 and $1600 \mathrm{~Hz}$. The wheel component dominates the noise above $2 \mathrm{kHz}$, which remains invariant in the different predictions.

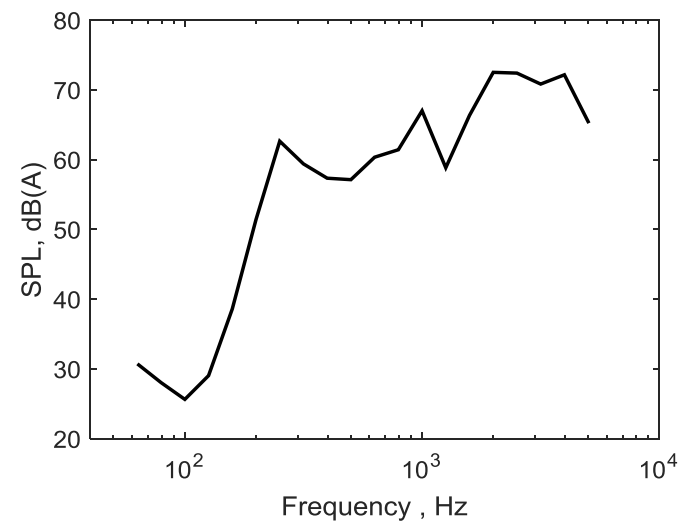

Figure 11 Predicted A-weighted sound pressure level of the component from the wheels

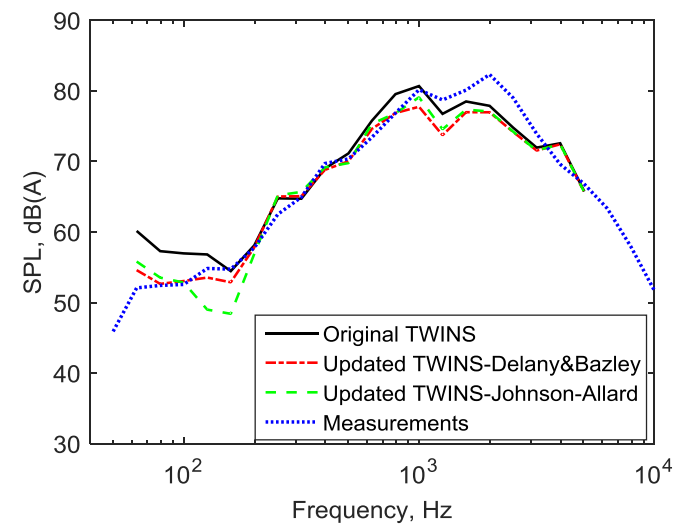

Figure 12 Comparison of A-weighted sound pressure spectra between different predictions and the measurements

\section{Conclusions}

A new model has been proposed to predict the sound radiation from the track using the boundary element method, taking account of the effects of the ground, including the ballast. Measurements have been performed of the flow resistivity, porosity and diffuse field absorption of 1:5 scale ballast. These are used with the Johnson-Allard model to represent the acoustic impedance. Good agreement can be found between the predicted absorption and the corresponding measurement results. The Delany and Bazley model is also used with a higher value of flow resistivity to obtain an alternative impedance for use in the numerical models. By taking the ballast absorption into account, it is found that the sound radiation from the rails and the sleepers are significantly affected, especially at low frequency. The models have been validated by measuring the sound radiation from a 1:5 scale track and comparing the results with the predictions. It is found that using the impedance derived from the Johnson-Allard model in the BEM models does not give satisfactory results, which is probably due to the need to account for extended reaction in the ballast layer. On the other hand using the Delany and Bazley model with a higher value of flow resistivity gives more satisfactory results. The models have finally been used with the TWINS software to predict the sound radiation from an operational track and comparisons have been made with field measurements. Differences are introduced in the sound radiation components predicted for the rails and the sleepers, particularly at low frequency. Good agreement is seen with field measurements. The new models are found to give an improvement at low frequencies, where the sleeper is the dominant noise source.

\section{Acknowledgements}

The work described here has been supported by the EPSRC under the programme grants EP/H044949/1, 'Railway Track for the $21^{\text {st }}$ Century (Track 21)' and EP/M025276/1, 'The science and analytical tools to design long life, low noise railway track systems (Track to the Future)'. 
All data published in this paper are openly available from University of Southampton repository at $10.5258 / \mathrm{SOTON} / \mathrm{D} 0013$.

\section{References}

1. Thompson, D.J., Hemsworth, B., Vincent, N.: Experimental validation of the TWINS prediction program for rolling noise, Part 1: description of the model and method, Journal of Sound and Vibration. 193, 123-135 (1996).

2. Thompson, D.J., Fodiman, P., Mahé, H.: Experimental validation of the TWINS prediction program for rolling noise, part 2: results, Journal of Sound and Vibration. 193, 137-147 (1996).

3. Thompson, D.J., Janssens, M.H.A.: Track Wheel Interaction Noise Software, Version 3.0, Theoretical manual, TPD-HAG-MEMO-960343. 1996.

4. Zhang, X., Squicciarini, G., Thompson, D.J.: Sound radiation of a railway rail in close proximity to the ground, Journal of Sound and Vibration. 362, 111-124 (2016).

5. Zhang, X., Thompson, D.J., Squicciarini, G.: Sound radiation of railway sleepers, Journal of Sound and Vibration. 369, 178-194 (2016).

6. Broadbent, R.A., Thompson, D.J., Jones, C.J.C.: The acoustic properties of railway ballast. Euronoise 2009, Edinburgh.

7. Attenborough, K., Boulanger, P., Qin, Q., Jones, R.: Predicted influence of ballast and porous concrete on rail noise, Internoise 2005. Brazil.

8. BS EN 13450: 2013.: Aggregates for railway ballast.

9. Zhang, X., Thompson, D.J., Squicciarini, G.: Effects of railway ballast on the sound radiation from the sleepers, Euronoise 2015. Maastricht (Netherlands).

10. Johnson, D.L., Koplik, J., Dashen, R.: Theory of dynamic permeability and tortuosity in fluid-saturated porous media, Journal of Fluid Mechanics. 176, 379-402 (1987).

11. Allard, J.F., Atalla, N.: Propagation of Sound in Porous Media: modelling sound absorbing materials, Wiley, Chichester, 2009.

12. Delany, M.E., Bazley, E.N.: Acoustical properties of fibrous absorbent materials, Applied Acoustics. 3, 105-116 (1970).

13. Heutschi, K.: Sound propagation over ballast surfaces, Acta Acustica United With Acustica. 95, 1006-1012 (2009).

14. Thompson, D.J.: Railway noise and vibration mechanisms, modelling and means of control. Elsevier, 2008.

15. Grassie, S. L., Gregory, R. W., Harrison, D., Johnson, K. L.: The dynamic response of railway track to high frequency vertical excitation, Journal of Mechanical engineering science. 24, 77-90 (1982).

16. EN 15461:2008+A1:2010.: Railway applications. Noise emission. Characterization of the dynamic properties of track selections for pass by noise measurements.

17. Squicciarini, G., Toward, M.G.R., Thompson, D.J.: Experimental procedures for testing the performance of rail dampers, Journal of Sound and Vibration. 359, 21-39 (2015).

18. Fahy, F., Gardonio, P.: Sound and structural vibration: radiation, transmission and response. Second edition, Elsevier, Oxford, 2007.

19. Squicciarini, G., Thompson, D.J., Toward, M.G.R., Cottrell, R.A.: The effect of temperature on railway rolling noise, Journal of Rail and Rapid Transit. doi: 10.1177/0954409715614337, 2015. 